\title{
Growth Fitness of Indigenous Wine Yeasts in Grape Musts from Different Vitis Species
}

\section{María Laura Raymond Eder* and Alberto Luis Rosa}

Laboratorio de Genética y Biología Molecular, IRNASUS-CONICET, Facultad de

Ciencias Químicas, Universidad Católica de Córdoba, Córdoba, Argentina

*Corresponding Author: Maria Laura Raymond Eder, Laboratorio de Genética y

Biología Molecular, IRNASUS-CONICET, Facultad de Ciencias Químicas, Universidad

Católica de Córdoba, Córdoba, Argentina.
Received: February 26, 2021

Published: March 25, 2021

(C) All rights are reserved by María Laura

Raymond Eder and Alberto Luis Rosa.

\section{Abstract}

Yeast communities associated with Vitis vinifera L. niches (i.e. grapes and fermenting grape musts) have been widely characterized. Less is known, however, about yeast communities present in other non-vinifera Vitis ecosystems. Moreover, there are no studies concerning eventual must-specific growth fitness of indigenous wine yeast species. In this work, we have characterized the potential must-specific growth fitness of ten different indigenous wine yeast species (i.e. C. azymoides, C. californica, $H$. uvarum, $H$. vineae, $I$. hanoiensis, M. pulcherrima, P. cecembensis, T. delbrueckii, S. bacillaris and S. cerevisiae). All the analyzed strains were isolated from spontaneously fermenting musts of V. vinifera L. (cv. Malbec) and/or V. labrusca L. (cv. Isabella) grapes harvested from vineyards in a shared terroir. Yeast identification was performed using standard ITS-rDNA RFLPs and/or microsatellite genotyping. Growth fitness of selected yeast species and strains, on Malbec and Isabella pasteurized grape must media, was studied by measuring lag phases (i.e. Lag Time) and maximum growth rates (i.e. $\mu$ max). Results showed that rare yeast species isolated from the Isabella ecosystem (i.e. P. cecembensis and I. hanoiensis) have better growth parameters when growing in Isabella grape must. The growth parameters of other wine yeast species, isolated from Malbec and Isabella ecosystems, did not show any clear associations with their musts of origin. Our findings suggest that the presence of two rare yeast species in Isabella could result from their growth advantage to this Vitis ecosystem. It is possible that yeast communities assembled in alternative grape musts result from the growth fitness of yeast species and strains to each specific Vitis species. Non-conventional Vitis ecosystems may constitute a reservoir of unique yeast strains valuable in the winemaking industry.

Keywords: Vitis vinifera L.; Vitis labrusca L.; Grape Must; Yeast; Non-Saccharomyces; Saccharomyces cerevisiae; Microbial Fitness

\section{Introduction}

Spontaneously fermenting grape musts are complex biological and chemical ecosystems where a diverse community of microorganisms (i.e. yeast, bacteria and filamentous fungi) contributes to the final wine chemistry [1]. A rich diversity of nonSaccharomyces and $S$. cerevisiae wine yeast species predominate during alcoholic fermentations $[2,3]$. These dynamic microbiotas produce a large variety of secondary metabolites that defines the organoleptic profile of beverages [4]. Because of its scientific and industrial relevance, the study of the indigenous microbial communities in grapes and fermenting grape musts ecosystems constitutes a major research area in oenology [2,5-8].

The identity and relative abundance of the various yeast species found at early stages of must fermentation appear to be 
determined by biotic (e.g. general microbiota, killer factors, grape variety, etc.) and abiotic (e.g. pH, temperature, osmotic pressure, ethanol, total nitrogen, $\mathrm{SO}_{2}$, etc.) factors [9-11]. A common pattern of development of certain wine yeast species has been recognized in spontaneously fermenting musts from Vitis vinifera L. grapes, being non-Saccharomyces the most common yeast species at initial stages $[2,5,12-14]$ and $S$. cerevisiae the dominant species at the middle and final stages of fermentation [3].

Extensive research has been conducted on the complexity and fermentation-driven dynamics of yeast communities in $V$. vinifera L. ecosystems $[2,4]$. However, only a few studies have examined the yeast communities in non-vinifera Vitis ecosystems [15]. These non-conventional Vitis ecosystems may harbor a rich diversity of yeast species and strains with unique metabolic traits $[13,14]$. The diversity of yeast communities in grapes and grape musts from Vitis labrusca L. cultivars and its hybrids has been studied in vineyards from Brazil [16,17], the Azores Archipelago (Portugal) [18] and Argentina $[13,14]$. These studies highlighted the remarkable diversity of non-Saccharomyces yeast species in these particular non-conventional Vitis ecosystems, also suggesting the existence of specific Vitis-yeast species associations [13,14].

In this work, we characterized fitness parameters of indigenous yeasts isolated from two different Vitis ecosystems. Yeast growth studies were performed using musts from Malbec ( $V$. vinifera L.) and Isabella (V. labrusca L.) grapes harvested from neighboring vineyards. This experimental design allowed us to explore the growth fitness of indigenous wine yeast strains in grape musts from different neighbor Vitis ecosystems.

\section{Materials and Methods}

Malbec (V. vinifera L.) and Isabella (V. labrusca L.) grape musts

Malbec (Vitis vinifera L.) and Isabella (Vitis labrusca L.) grapes were harvested at their optimal ripeness stages from several

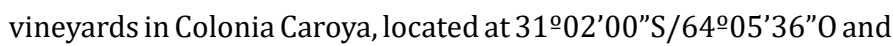
491 meters above sea level, in the province of Córdoba, Argentina, during the 2018 vintage. The region has an annual rainfall of 765 $\mathrm{mm}$ and a mean temperature of $15.8^{\circ} \mathrm{C}$. Separate must samples were taken immediately after grape crushing from tanks located in a room of a local winery not previously used for winemaking. Grape musts were transported under refrigeration to the laboratory, and $1 \mathrm{l}$ of each must was filtered and centrifuged at $3000 \mathrm{rpm}$ for 10 min at room temperature. Supernatants were pasteurized in 180 $\mathrm{ml}$ aliquots in a water bath $\left(70^{\circ} \mathrm{C}, 30 \mathrm{~min}\right)$ and stored at $-20^{\circ} \mathrm{C}$ for growth analyses. Physicochemical analyses of the musts (i.e. ${ }^{\circ} \mathrm{Brix}$, $\alpha$ amino, ammonium, yeast assimilable nitrogen -YAN-, $\mathrm{pH}$, total acidity, volatile acidity and density) were performed following standard oenological procedures [14].

\section{Indigenous yeast strains}

Yeast strains used in this study were isolated from spontaneously fermenting musts of $V$. vinifera L. and $V$. labrusca L. grapes, as previously described $[13,14]$. Additionally, the commercial Saccharomyces cerevisiae yeast strain Lalvin EC1118 (Lallemand, Canada) was included as a control. Strains used in this study are indicated in table 1 . Yeasts were previously identified to species level by PCR-RFLP (Table 2) and/or DNA sequencing of their 5.8ITS (Internal Transcribed Spacer) rDNA regions, using ITS1 and ITS4 primers $[13,14]$. 5.8-ITS sequences were deposited in the NCBI GenBank database under the accession numbers KY693700 (Candida azymoides: IT0-016), KY693709 (Candida californica: IT2010), VG734839 (Hanseniaspora uvarum: IT117-013), VG734841 (Hanseniaspora uvarum: MT017-035), KY693711 (Hanseniaspora vineae: IT2-021), KY693701 (Issatckenkia hanoiensis: IT0-025), KY693704 (Pichia cecembensis: IT0-042), MG734849 (Starmerella bacillaris: MT017-001), KY693706 (S. bacillaris: IT1-033), MG734853 (S. cerevisiae: MT217-023), MG734858 (S. cerevisiae: IT217-022), and KY693707 (T. delbrueckii: IT1-039). Furthermore, $S$. cerevisiae and $S$. bacillaris isolates were characterized to strain level by microsatellite genotyping $[19,20]$.

\section{Yeast growth analyses in alternative grape musts}

To assess yeast growth in alternative media, selected strains corresponding to ten different yeast species (Table 1), were cultivated in Malbec ( $V$. vinifera L.) and Isabella (V. labrusca L.) grape musts as well as YPD [yeast extract $1.0 \%(\mathrm{w} / \mathrm{v})$, peptone $2.0 \%(\mathrm{w} / \mathrm{v})$, glucose $2.0 \%$ ] media. Yeast strains were grown in 100well honeycomb plates (Growth Curves, USA) filled with $200 \mu \mathrm{l}$ of filtered and pasteurized grape must or sterile YPD media. Yeasts were pre-cultivated in YPD media for $16 \mathrm{~h}$ at $25^{\circ} \mathrm{C}$ and inoculated into the grape musts or liquid YPD to a final concentration of 1 x $10^{6}$ cells $/ \mathrm{ml}$. Growth was monitored by $\mathrm{OD}_{600}$ measurements for 48 hours at $25^{\circ} \mathrm{C}$, with constant agitation, using a microplate spectrophotometer (Bioscreen C(C). The well position on the microplate was randomized and four replicates were run for each strain and growth media condition. 


\begin{tabular}{|l|c|c|c|}
\hline Yeast species & Must $^{\mathbf{1}}$ & Year $^{\mathbf{2}}$ & Isolate \\
\hline \multirow{3}{*}{ S. cerevisiae } & Commercial & - & EC1118 \\
\cline { 2 - 4 } & Malbec & 2017 & MT217-023 \\
\cline { 2 - 4 } S. bacillaris & Isabella & 2016 & IT1217-022 \\
\hline \multirow{3}{*}{ T. delbrueckii } & Malbec & 2017 & MT017-001 \\
\cline { 2 - 4 } & Isabella & 2016 & IT1-033 \\
\hline \multirow{2}{*}{ H. uvarum } & Malbec & 2017 & MT017-059 \\
\hline \multirow{2}{*}{ H. vineae } & Isabella & 2016 & IT1-039 \\
\cline { 2 - 4 } & Malbec & 2017 & MT017-035 \\
\hline \multirow{2}{*}{ C. azymoides } & Isabella & 2017 & IT117-013 \\
\cline { 2 - 4 } & Isabella & 2016 & IT2-021 \\
\hline M. pulcherrima & Malbec & 2017 & MT017-061 \\
\hline C. californica & Isabella & 2016 & IT0-016 \\
\hline I. hanoiensis & Isabella & 2016 & IT2-010 \\
\hline P. cecembensis & Isabella & 2016 & IT0-042 \\
\hline
\end{tabular}

Table 1: Analyzed yeast strains.

1: Spontaneously fermenting must; ${ }^{2}$ : Year of isolation.

\begin{tabular}{|l|c|c|c|}
\hline \multirow{2}{*}{ Yeast species } & PCR product & \multicolumn{2}{|c|}{ RFLP ${ }^{\mathbf{1}}$ pattern } \\
\cline { 3 - 4 } & (size; bp) & CfoI & HinfI \\
\hline S. cerevisiae & 850 & $388+359+141$ & $373+375+120$ \\
\hline S. bacillaris & 460 & $\begin{array}{c}56+103+105+ \\
196\end{array}$ & $225+235$ \\
\hline T. delbrueckii & 800 & $\begin{array}{c}359+243+159 \\
+108\end{array}$ & $473+400$ \\
\hline H. uvarum & 770 & $359+340+116$ & $376+210+178$ \\
\hline H. vineae & 754 & $\begin{array}{c}270+156+143 \\
+93\end{array}$ & $389+373$ \\
\hline C. azymoides & 480 & $213+206+80$ & $257+206$ \\
\hline M. pulcherrima & 404 & $233+106+93$ & $222+196$ \\
\hline C. californica & 482 & $238+109+71$ & $269+225$ \\
\hline I. hanoiensis & 472 & $137+98+86+66$ & $272+228$ \\
\hline P. cecembensis & 496 & $256+116+64$ & $277+129$ \\
\hline
\end{tabular}

Table 2: DNA digestion patterns of ITS-5.8S regions of various yeast species.

1: Restriction fragment length polymorphism (i.e. bp).
Data analysis of growth measurements

Data from the microplate reader were transformed with the polynomial curve $\mathrm{y}=-0.0018^{*} \mathrm{x} 3+0.1464^{*} \mathrm{x} 2+0.7757^{*} \mathrm{x}+$ 0.0386 to correct the non-linearity of the optical recording at higher cell densities as previously reported [21]. Growth kinetic data were fitted using the Richards flexible inflection point model implemented by the fit growth model function, R package growth rates. This model allows the estimation of the maximal growth rate ( $\mu$ max). A second parameter, Lag Time, was manually computed from raw data by considering the time necessary to reach twice the $\mathrm{OD}_{600}$ of the inoculums [19]. The following full linear model was applied for estimating the effects of the must of isolation and the growth media and their possible interactions: $\mathrm{Lm} 1:$ Yik $=\mathrm{m}+\mathrm{Im} \mathrm{i}$ $+\mathrm{Gm} \mathrm{k}+(\mathrm{Im}: \mathrm{Gm}) \mathrm{ik}+\mathrm{E}$ ijk, where $\mathrm{Y}$ is the value of the trait ( $\mu$ max and Lag Time), $\mathrm{m}$ is the overall mean, for $\mathrm{j}$ : Im (isolation must, $\mathrm{i}=1$ to 2), and $\mathrm{Gm}$ (growth media, $\mathrm{k}=1$ to 3 ), and $\mathrm{E}$ is the residual error. Homoscedasticity of the ANOVA was tested by Levene Test function and visual inspection of dispersion diagrams (car package), while the normal distribution of models' residuals was estimated by visual inspection (qq plot). Comparisons between treatments were performed by Tukey test (Infostat; Universidad Nacional de Córdoba).

\section{Results}

Indigenous yeasts in spontaneously fermenting musts of grapes harvested in neighbor Vitis vinifera L. and Vitis labrusca L. ecosystems

Remarkable differences have been recognized in the diversity and identity of non-Saccharomyces species isolated from $V$. vinifera L. and V. non-vinifera ecosystems (See Raymond Eder and Rosa, 2019). In this work we characterized a collection of indigenous yeast isolates from spontaneously fermenting musts of Malbec (V. vinifera L.) and Isabella (V. labrusca L.) grapes harvested from neighbor vineyards (see Materials and Methods section). Table 2 shows the ITS-rDNA RFLP patterns of some rare wine yeasts, used to establish species identity. ITS-rDNA PCR products were also sequenced to further verify the identity of the species analyzed in this work (See materials and methods section).

Among the isolates shown in table 1, some yeast species rarely isolated from $V$. vinifera $L$. fermenting grape musts can be identified (i.e. Candida azymoides, Candida californica, Issatchenkia hanoiensis and Pichia cecembensis). A comparison of the large yeast 
biodiversity present at early stages of the Malbec and Isabella spontaneously fermenting grape musts [14] is shown in figure 1. Previous microsatellite loci genotyping studies of a large number of strains from a representative non-Saccharomyces yeast species (i.e. S. bacillaris) and S. cerevisiae, isolated from Malbec and Isabella ecosystems [19,22], also revealed a great biodiversity of strains of these yeast species. Taken together, these results highlight the remarkable biodiversity and presence of extraordinary wine yeasts in the non-vinifera Vitis ecosystem Isabella.

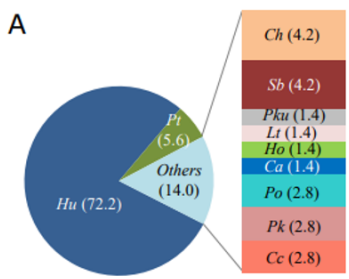

V. labrusca L.
B

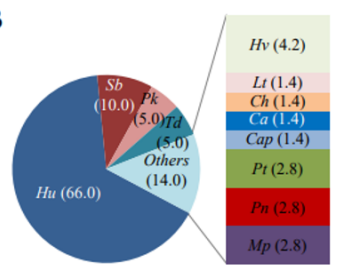

V. vinifera L.
Figure 1: Diversity of non-Saccharomyces species isolated from spontaneously fermenting must of $V$. vinifera $L$. and $V$. labrusca L. grapes harvested in a shared terroir. Relative contribution of non-Saccharomyces yeast species representing more than $1 \%$ of the isolates from spontaneously fermenting V. labrusca L. (A) and V. vinifera L. (B) grape musts (vintage 2017). Numbers in parentheses indicate percentages. Non-Saccharomyces species are: Ca (Candida azymoides), Cap (Candida apicola), Cc (Candida californica), Ch (Candida hellenica), Hu (Hanseniaspora uvarum), Hv (Hanseniaspora vineae), Lt (Lachancea thermotolerans), Mp (Metschnikowia pulcherrima), Pc (Pichia cecembensis), Pk

(Pichia kluyveri), Pku (Pichia kudriavzevii), Pn (Pichia norvegensis), Po (Pichia occidentalis), Pt (Pichia terricola), Sb

(Starmerella bacillaris) and Td (Torulaspora delbrueckii).

Growth fitness of indigenous yeasts in Malbec and Isabella grape musts

The remarkable yeast biodiversity observed in neighboring Vitis ecosystems (i.e. V. vinifera L. and V. labrusca L.) from the same geographic region (Table 1 and figure 1) suggested that specific traits of different Vitis species could contribute to structure their associated, specific yeast communities (i.e. yeast species and/or strains) [23].

To address the above mentioned hypotheses, in this work we explored the growth phenotypes of a subset of 16 indigenous yeast strains corresponding to ten species isolated from Malbec and Isabella fermenting grape musts (Table 1). We hypothesized that yeast strains isolated from a specific Vitis grape must could have a competitive advantage when grown in their must ecosystem of origin. In these studies, growth fitness in pasteurized Malbec and Isabella grape musts was assessed for each strain (See materials and methods section). The fitted $\mathrm{OD}_{600}$ growth data (Figure 2) allowed the estimation of the maximum growth rate (i.e. $\mu$ max) and the Lag phase (i.e. Lag Time) was also calculated using the growth raw data (Figure 3). The effect of the original must of isolation and the tested must for growth was estimated by two-way ANOVA (model Lm1). Table 3 shows that the growth media significantly impacted the two parameters (i.e. $\mu$ max and Lag Time) in most of the strains tested, explaining much of their variance.
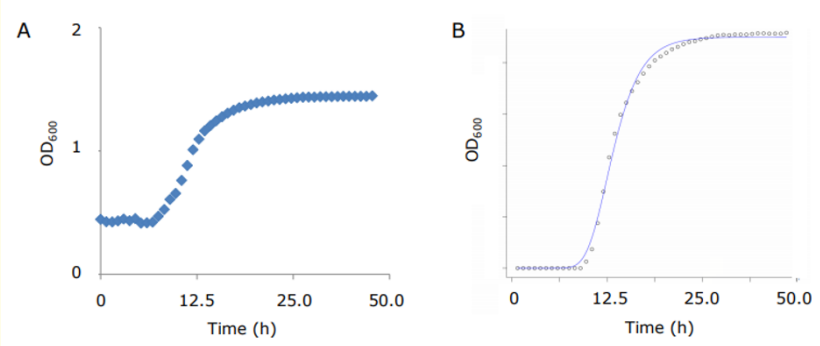

Figure 2: Fitting of growth kinetic data. Example of the analysis of $S$. bacillaris IT1-033 growth in Malbec grape must. (A) Raw $\mathrm{OD}_{600}$ growth data. (B) Fitted $\mathrm{OD}_{600}$ data using the Richards flexible inflection point model. The fit of the model is shown as a continuous blue line.

Tukey's analyses of the data (Table 4) showed that I. hanoiensis, originally isolated from the Isabella ecosystem, showed better growth parameters (i.e. shorter Lag time and higher $\mu$ max) when grown in Isabella grape must. Interestingly, although the growth must (i.e. Malbec or Isabella) did not impart a significant difference in the Lag phase of $P$. cecembensis, a remarkable higher maximum 


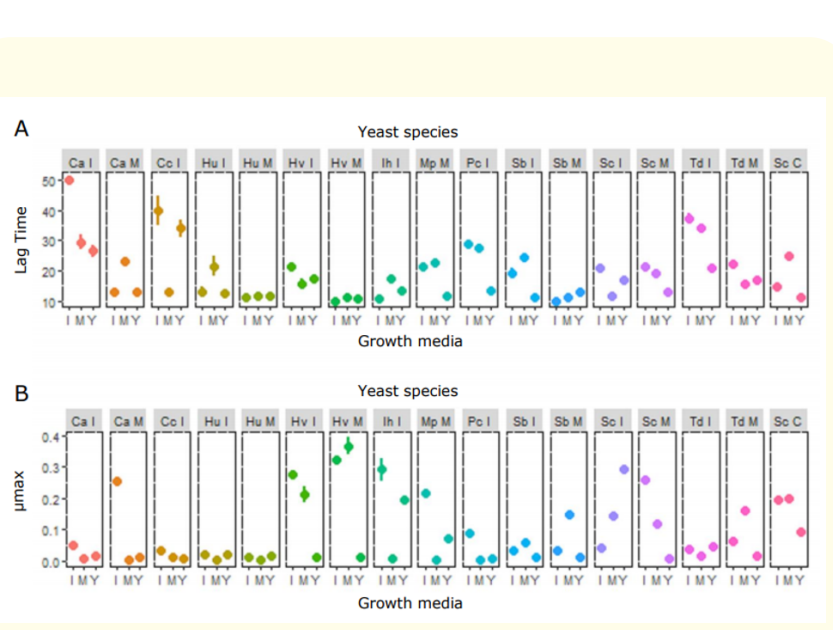

Figure 3: Growth of indigenous yeast species in Malbec, Isabella and YPD media. (A) Mean of the Lag Times (in hours) of each strain in the alternative Malbec (M), Isabella (I) and YPD

(Y) growth media. (B) Mean of the $\mu$ max of each strain in the alternative Malbec (M), Isabella (I) and YPD (Y) growth media. Ca I (C. azymoides, Isabella isolate), Ca M (C. azymoides, Malbec isolate), Cc I (C. californica, Isabella isolate), Hu I (H. uvarum, Isabella isolate), Hu M (H. uvarum, Malbec isolate), $\mathrm{Hv}$ I ( $H$. vineae, Isabella isolate), $\mathrm{Hv} \mathrm{M}$ (H. vineae, Malbec isolate), Ih I (I. hanoiensis, Isabella isolate), Mp M (M. pulcherrima, Malbec isolate), Pc I (P. cecembensis, Isabella isolate), Sb I (S. bacillaris,

Isabella isolate), $\mathrm{Sb} \mathrm{M}$ (S. bacillaris, Malbec isolate), Sc I ( $S$. cerevisiae, Isabella isolate), Sc M (S. cerevisiae, Malbec isolate), Td I (T. delbrueckii, Isabella isolate), Td M (T. delbrueckii, Malbec isolate) and Sc C (S. cerevisiae, Commercial isolate).

growth rate for this strain was observed in Isabella must (Table 4). Growth parameters of other yeast species, either rarely or ubiquitously recognized in Malbec and/or Isabella ecosystems, were significantly affected by the interactions between the two analyzed factors (Table 3). For example, C. californica, a yeast species specifically associated with the Isabella ecosystem, showed a better growth parameter (i.e. lower Lag phase) in Malbec must. For C. azymoides, the strain isolated from Malbec revealed a fastest growth in the Isabella must, while the Isabella isolate did not show differences in the Lag phases in both musts. Finally, the Malbec isolates from T. delbrueckii and S. bacillaris showed lower Lag Times in both grape musts than the Isabella isolates. Other yeast

\begin{tabular}{|c|c|c|c|c|c|c|c|}
\hline \multirow{2}{*}{$\begin{array}{c}\text { Yeast } \\
\text { species }\end{array}$} & \multicolumn{2}{|c|}{ Isolation must } & \multicolumn{2}{|c|}{$\begin{array}{c}\text { Growth } \\
\text { media }\end{array}$} & \multicolumn{2}{|c|}{$\mathbf{I m}^{*} \mathbf{G m}^{1}$} & \\
\hline & Effect & $\begin{array}{c}\text { p- } \\
\text { value }\end{array}$ & Effect & $\begin{array}{c}\text { p- } \\
\text { value }\end{array}$ & Effect & $\begin{array}{c}\text { p- } \\
\text { value }\end{array}$ & \\
\hline S. cerevisiae & na & ns & 16.7 & . & 52.4 & $* * *$ & \multirow{10}{*}{ Lag Time } \\
\hline S. bacillaris & 33.9 & $* * *$ & 16.9 & $*$ & 37.3 & $* * *$ & \\
\hline T. delbrueckii & 50.9 & $* * *$ & 21.6 & $*$ & 11.8 & . & \\
\hline H. uvarum & na & ns & na & ns & na & ns & \\
\hline H. vineae & 62.5 & $* * *$ & na & ns & na & ns & \\
\hline C. azymoides & 48.8 & $* * *$ & 9.0 & . & 13.5 & $*$ & \\
\hline $\begin{array}{l}\text { M. pulcher- } \\
\text { rima }\end{array}$ & na & na & 82.6 & . & na & na & \\
\hline C. californica & na & na & 75.4 & . & na & na & \\
\hline I. hanoiensis & na & na & 58.3 & . & na & na & \\
\hline $\begin{array}{l}\text { P. cecemben- } \\
\text { sis }\end{array}$ & na & na & 82.4 & . & na & na & \\
\hline S. cerevisiae & 10.1 & $* * *$ & 12.3 & $* * *$ & 59.8 & $* * *$ & \multirow{10}{*}{$\mu \max$} \\
\hline S. bacillaris & na & ns & 78.6 & $* * *$ & na & ns & \\
\hline T. delbrueckii & 13.7 & . & na & ns & 44.1 & $*$ & \\
\hline H. uvarum & na & ns & 58.8 & $* * *$ & na & ns & \\
\hline H. vineae & 0.7 & . & 97.8 & $* * *$ & na & ns & \\
\hline C. azymoides & na & ns & 91.1 & $* * *$ & na & ns & \\
\hline $\begin{array}{l}\text { M. pulcher- } \\
\text { rima }\end{array}$ & na & na & 98.2 & $* * *$ & na & na & \\
\hline C. californica & na & na & 56.5 & . & na & na & \\
\hline I. hanoiensis & na & na & 93.8 & $* * *$ & na & na & \\
\hline $\begin{array}{l}\text { P. cecemben- } \\
\text { sis }\end{array}$ & na & na & 93.9 & $* * *$ & na & na & \\
\hline \multicolumn{8}{|c|}{$\begin{array}{l}{ }^{1} \text { Isolation must (Im) and Growth media }(\mathrm{Gm}) \text { interactions; } \\
\text { significance levels: }{ }^{(* * *)}<0.0001 ;^{\left(* *^{\prime}<0.001 ;^{(*)}<0.01 ;\right.}{ }^{\prime .}<0.05 \text {; ns: } \\
\text { not significant; na: not applicable. }\end{array}$} \\
\hline
\end{tabular}

Table 3: Analyses of variance of isolation must and growth media interactions for Lag time and $\mu$ max.

species (i.e. H. uvarum and $H$. vineae) did not show significant differences in the growth parameters for the interactions between the two factors analyzed (Table 3).

Physicochemical analyses of the Malbec and Isabella grape musts

Results from the physicochemical analyses of the Malbec and Isabella grape musts are shown in table 5 . As it was previously 


\begin{tabular}{|c|c|c|c|c|c|c|c|}
\hline \multirow{3}{*}{$\begin{array}{c}\text { Yeast } \\
\text { species }\end{array}$} & \multirow{3}{*}{$\begin{array}{l}\text { Isolation } \\
\text { must }\end{array}$} & \multicolumn{3}{|c|}{ Growth media $^{1}$} & \multirow{2}{*}{\multicolumn{3}{|c|}{$\mu \max$}} \\
\hline & & \multicolumn{3}{|c|}{ Lag Time } & & & \\
\hline & & $\mathbf{M}$ & I & $\mathbf{Y}$ & $\mathbf{M}$ & I & $\mathbf{Y}$ \\
\hline \multirow{3}{*}{ S. cerevisiae } & Commercial & e & abcd & $\mathrm{a}$ & def & def & c \\
\hline & Malbec & bcde & de & $\mathrm{abc}$ & $\mathrm{cd}$ & ef & $\mathrm{a}$ \\
\hline & Isabella & $\mathrm{ab}$ & cde & abcde & cde & $\mathrm{b}$ & $\mathrm{f}$ \\
\hline \multirow{2}{*}{ S. bacillaris } & Malbec & $\mathrm{a}$ & $\mathrm{a}$ & $\mathrm{ab}$ & \multirow{2}{*}{\multicolumn{3}{|c|}{ na }} \\
\hline & Isabella & $\mathrm{c}$ & bc & $\mathrm{a}$ & & & \\
\hline \multirow{2}{*}{ T. delbrueckii } & Malbec & $\mathrm{a}$ & $\mathrm{a}$ & $\mathrm{a}$ & $\mathrm{ab}$ & $\mathrm{ab}$ & $a b$ \\
\hline & Isabella & $\mathrm{b}$ & $\mathrm{b}$ & a & $\mathrm{b}$ & $\mathrm{ab}$ & $a b$ \\
\hline \multirow{2}{*}{ C. azymoides } & Malbec & $\mathrm{b}$ & $\mathrm{a}$ & $\mathrm{a}$ & \multirow{2}{*}{\multicolumn{3}{|c|}{ na }} \\
\hline & Isabella & $\mathrm{bc}$ & $\mathrm{c}$ & $\mathrm{bc}$ & & & \\
\hline $\begin{array}{l}\text { M. pulcher- } \\
\text { rima }\end{array}$ & Malbec & $\mathrm{b}$ & $\mathrm{b}$ & a & $\mathrm{a}$ & c & $\mathrm{b}$ \\
\hline C. californica & Isabella & $\mathrm{a}$ & $\mathrm{b}$ & $\mathrm{b}$ & $\mathrm{ab}$ & $\mathrm{b}$ & $\mathrm{a}$ \\
\hline I. hanoiensis & Isabella & $\mathrm{b}$ & $\mathrm{a}$ & $\mathrm{ab}$ & $\mathrm{a}$ & $\mathrm{b}$ & $\mathrm{b}$ \\
\hline $\begin{array}{l}\text { P. cecemben- } \\
\text { sis }\end{array}$ & Isabella & $\mathrm{b}$ & $\mathrm{b}$ & $\mathrm{a}$ & $\mathrm{a}$ & c & $\mathrm{b}$ \\
\hline
\end{tabular}

${ }^{1} \mathrm{M}$, Malbec grape must; I, Isabella grape must; Y, YPD media. Tukey analyses for strains isolated from only one must are shown in gray. Values with a common letter are not significantly different ( $p$ > 0.05); mean values: $a<f$. na: not applicable.

Table 4: Tukey analyses of isolation must and growth media interactions for Lag time and $\mu$ max.

described [14], the initial content of reducing sugars ( ${ }^{\circ}$ Brix) was higher in the Malbec must than in the Isabella grape must.
Furthermore, the Malbec and Isabella $\mathrm{pH}$ values were consistent with previous analyses $[13,14]$. The greatest differences were observed among the content of nitrogen compounds in the musts (i.e. $\alpha$ Amino, Ammonium, and Yeast Assimilable Nitrogen -YAN-), which were higher for the Isabella musts than for the Malbec musts analyzed.

\section{Discussion and Conclusion}

There is extensive research on the characterization of the microbiological communities associated with Vitis vinifera L. grapes and musts that contribute to the fermentation process. Grape varieties themselves could condition the assembly and dynamics of the microbial population during spontaneous fermentation [2426]. A more diverse non-Saccharomyces and S. cerevisiae strain population has been recognized in vineyards having several grape varieties than vineyards cultivating only one grape variety $[25,26]$. This observation suggests that vineyards harboring multiple Vitis species may show a more diverse yeast community as compared to vineyards with a single Vitis species. Thus, specific structural and/or general physicochemical factors of each grape varietal may influence the structure and fitness of their specific grape yeast microbiota [27]. Interestingly, non-vinifera Vitis species, a poorly characterized microbial niche, harbor wine yeast species rarely recognized in the conventional $V$. vinifera L. ecosystem $[13,14]$.

In this study, we hypothesized that specific growth fitness of indigenous strains could be the result of a growth adaptive advantage to their Vitis ecosystem of origin. To explore this idea, we characterized the growth fitness of indigenous yeast species,

\begin{tabular}{|l|c|c|c|c|c|c|c|c|}
\hline \multicolumn{1}{|c|}{ Must } & ${ }^{\circ} \mathbf{B r i x}$ & $\begin{array}{c}\boldsymbol{\alpha} \text { Amino } \\
(\mathbf{m g} / \mathbf{l})\end{array}$ & $\begin{array}{c}\mathbf{N H}_{\mathbf{4}}{ }^{+} \\
\mathbf{( m g / l )}\end{array}$ & $\begin{array}{c}\text { YAN } \\
(\mathbf{m g} / \mathbf{l})\end{array}$ & $\mathbf{p H}$ & $\begin{array}{c}\text { Total acidity } \\
\mathbf{( g / l )}\end{array}$ & $\begin{array}{c}\text { Volatile } \\
\text { acidity (g/l) }\end{array}$ & $\begin{array}{c}\text { Density } \\
\mathbf{( g / m )}\end{array}$ \\
\hline Malbec & 21.4 & 45.3 & 37.9 & 76.38 & 3.74 & 2.5 & 0.18 & 1.089 \\
\hline Isabella & 18.4 & 104.9 & 62.5 & 156.18 & 3.56 & 3.1 & 0.17 & 1.080 \\
\hline
\end{tabular}

Table 5: Chemical analyses of Malbec and Isabella grape musts.

isolated from $V$. vinifera $L$. and $V$. labrusca $L$. fermenting grape musts, on grape must from these alternative Vitis ecosystems. Relevant growth parameters of sixteen indigenous yeast isolates, corresponding to ten different wine yeast species, were determined in pasteurized Malbec and Isabella grape musts. As a control of yeast growth, all the studies were also performed in a nitrogen rich, limited in reducing sugars (i.e. glucose), medium (i.e. YPD). This medium served as a reference for the growth of all the yeast strains tested. $\mathrm{OD}_{600}$ growth data were fitted using the Richards flexible inflection point model to determine the maximum growth rate (i.e. $\mu$ max) parameter. Lag Times were also estimated by considering the time necessary to reach twice the $\mathrm{OD}_{600}$ of the inoculums. 
Our studies showed that I. hanoiensis and P. cecembensis isolates, rare yeast species preferentially associated with the Isabella ecosystem [13], have higher $\mu$ max values when growing in Isabella grape must. From this limited evidence, it is tempting to speculate that the presence of some rare yeast species in the Isabella ecosystem could result from their selective growth advantage to this Vitis species. The evaluation of the growth parameters of C. azymoides, C. californica, $H$. uvarum, $H$. vineae, M. pulcherrima, T. delbrueckii, $S$. bacillaris and $S$. cerevisiae indigenous strains, on the other hand, did not show a defined fitness preference for their corresponding Vitis ecosystem of isolation. Further studies are required to identify the factors of the Isabella grape musts specifically favoring the growth of I. hanoiensis and P. cecembensis yeast species.

\section{Author Contributions}

MLRE and ALR equally contributed to the conception, drafting, revising and final approval of the manuscript.

\section{Acknowledgements}

We thank S. Lauret and C. Reynoso (Bodega La Caroyense, Córdoba, Argentina) for Isabella and Malbec grape must samples and N. Litterio for the use of the microplate spectrophotometer Bioscreen C(C).

\section{Funding}

This work was supported by PICT-2014-3113 from FONCYT (Argentina) and SUV2015 (UCC) to ALR. ML Raymond Eder had a Fellowship from CONICET-Argentina. AL Rosa is Principal Investigator of CONICET-Argentina.

\section{Conflict of Interest}

The authors declare no conflicts of interest.

\section{Bibliography}

1. Fleet G H. "Wine yeasts for the future". FEMS Yeast Research 8.7 (2008): 979-995.

2. Jolly N P., et al. "Not your ordinary yeast: Non-Saccharomyces yeasts in wine production uncovered". FEMS Yeast Research 14.2 (2014): 215-237.

3. Albergaria H., et al. "Dominance of Saccharomyces cerevisiae in alcoholic fermentation processes: role of physiological fitness and microbial interactions". Applied Microbiology and Biotechnology 100.5 (2016): 2035-2046.

4. Fleet G. H. "Yeast interactions and wine flavour". International Journal of Food Microbiology 86.1-2 (2003): 11-22.

5. Padilla B., et al. "Yeast biodiversity from DOQ priorat uninoculated fermentations". Frontiers in Microbiology 7 (2016): 1-11.

6. Varela C. "The impact of non-Saccharomyces yeasts in the production of alcoholic beverages". Applied Microbiology and Biotechnology 100.23 (2016): 9861-9874.

7. Varela C., et al. "Yeasts found in vineyards and wineries". Yeast (2016).

8. Morgan H H., et al. "The grapevine and wine microbiome: Insights from high-throughput amplicon sequencing". Frontiers in Microbiology 8 (2017).

9. Ciani M., et al. "Yeast interactions in inoculated wine fermentation". Frontiers in Microbiology 7 (2016): 1-7.

10. Jolly N P., et al. "The role and use of non-Saccharomyces yeasts in wine production". South African Journal of Enology and Viticulture 27.1 (2017).

11. Masneuf-Pomarede I., et al. "The genetics of non-conventional wine yeasts: Current knowledge and future challenges". Frontiers in Microbiology 6 (2016).

12. Combina M., et al. "Yeasts associated to Malbec grape berries from Mendoza, Argentina". Journal of Applied Microbiology 98.5 (2005): 1055-1061.

13. Raymond Eder M L., et al. "Isolation and identification of the indigenous yeast population during spontaneous fermentation of Isabella (Vitis labrusca L.) grape must". Frontiers in Microbiology 8 (2017): 1-8.

14. Raymond Eder M L., et al. "Differences between indigenous yeast populations in spontaneously fermenting musts from $V$. vinifera L. and V. labrusca L. Grapes harvested in the same geographic location". Frontiers in Microbiology 9 (2018): 1-10.

15. Raymond Eder M L., et al. "Yeast diversity in Vitis non-vinifera ecosystems". Revista Argentina de Microbiologia 51.3 (2019): 278-283. 
16. Bezerra-Bussoli C., et al. "Yeast diversity isolated from grape musts during spontaneous fermentation from a brazilian winery". Current Microbiology 67.3 (2013): 356-361.

17. Cilião Filho M., et al. "Genetic diversity and pectinolytic activity of epiphytic yeasts from grape carposphere". Genetics and Molecular Research 16.2 (2017): 1-13.

18. Drumonde-Neves J., et al. "Yeast biodiversity in vineyard environments is increased by human intervention". PLOS ONE 11.8 (2016): 1-13.

19. Marullo P., et al. "SSU1 checkup, a rapid tool for detecting chromosomal rearrangements related to the SSU1 promoter in Saccharomyces cerevisiae: An ecological and technological study on wine yeast". Frontiers in Microbiology 11 (2020): 2020.04.23.055350.

20. Raymond Eder M. L., et al. "Vitis species, vintage, and alcoholic fermentation do not drive population structure in Starmerella bacillaris (synonym Candida zemplinina) species". Yeast 36.6 (2019).

21. Marti-Raga M., et al. "Nitrogen modulation of yeast fitness and viability during sparkling wine production". Food Microbiology 54 (2016): 106-114.

22. Raymond Eder M L., et al. "Vitis species, vintage, and alcoholic fermentation do not drive population structure in Starmerella bacillaris (synonym Candida zemplinina) species". Yeast 36.6 (2019): 411-420.

23. Raymond Eder M L. "Asociaciones específicas Vitis - microbiota de levaduras en ecosistemas enológicos". Universidad Católica de Córdoba (2021).

24. Bokulich N A., et al. "Microbial biogeography of wine grapes is conditioned by cultivar, vintage, and climate". Proceedings of the National Academy of Sciences of the United States of America 111.1 (2014): E139-148.

25. Cordero-Bueso G., et al. "Influence of the farming system and vine variety on yeast communities associated with grape berries". International Journal of Food Microbiology 145.1 (2011): 132-139.

26. Schuller D., et al. "Genetic diversity and population structure of Saccharomyces cerevisiae strains isolated from different grape varieties and winemaking regions". PLoS ONE 7.2 (2012).

27. Li S S., et al. "Yeast species associated with wine grapes in China”. International Journal of Food Microbiology 138.1-2 (2010): 85-90.

\section{Assets from publication with us}

- Prompt Acknowledgement after receiving the article

- Thorough Double blinded peer review

- Rapid Publication

- Issue of Publication Certificate

- High visibility of your Published work

Website: www.actascientific.com/

Submit Article: www.actascientific.com/submission.php

Email us: editor@actascientific.com

Contact us: +919182824667 